\title{
Influencing Experience: The Effects of Reading Game Reviews on Player Experience
}

\author{
Ian J. Livingston ${ }^{1}$, Lennart E. Nacke ${ }^{2}$, and Regan L. Mandryk ${ }^{1}$ \\ ${ }^{1}$ Department of Computer Science, University of Saskatchewan, \\ 110 Science Place, Saskatoon, Saskatchewan, Canada S7N 5C9 \\ ${ }^{2}$ Faculty of Business and Information Technology, University of Ontario Institute of \\ Technology, 2000 Simcoe St N, Oshawa, ON, Canada L1H 7K4 \\ firstname.lastname@usask.ca, Lennart.Nacke@acm.org \\ http://hci.usask.ca
}

\begin{abstract}
Game reviews are used by game developers for making business decisions and measuring the success of a title, and have been shown to affect player perception of game quality. We conducted a study where players read positive or negative reviews of a game before playing, and show that the valence of review text affected game ratings and that these differences could not be explained by mediating changes in mood. Although we show predictable changes in player experience over the course of the study (measured objectively through physiological sensors), there were no objective differences in experience depending on review valence. Our results suggest that reading reviews does not directly affect play experience, but rather is a post-play cognitive rationalization of the experience with the content of the review. Our results are important for understanding player experience and to the game industry where reviews and user forums affect a game's commercial success.
\end{abstract}

Keywords: Biasing effects, game reviews, critics, player experience, games, physiology, GSR, EMG, affect.

\section{Introduction}

Game reviews play a major role in the video game industry and in video game culture. They shape our understanding of games, sway business decisions and consumer choice, and impact player experience. The combination of these and other factors has made video game reviews a large and influential business. Players form opinions about a game before ever playing them due, in part, to the influence game reviews wield over other aspects of the business such as marketing, and also directly over players' purchase intent.

In our previous work [10] we demonstrated that biasing effects from reading negative game reviews cause players to rate the quality of a game significantly lower than after reading positive reviews, or reading no reviews at all. Our results showed that the changes in perceived game quality were not mediated by subjective changes in the players' overall moods. However, what remained unclear was whether the effect of game reviews caused a change in the player experience, or if we observed a 
post-play cognitive rationalization of player experience with the content of the review text. In the former situation, a player who read a negative review would enjoy the play experience less than a player who read a positive review. In the latter, both players would have a similar experience; however, the one who read a negative review would interpret their experience as having been more negative. This distinction is important as it helps us to understand the impact of reading game reviews on subsequent player experience in a time when it is common for players to read about a game prior to making a purchasing decision or before even playing a demo version of the game.

To determine whether player experience is directly affected by review text or whether the biasing effects are a cognitive rationalization of player experience with the content of the review, we designed an experiment that measures a player's subjective rating of a game, their subjective mood throughout the experiment, and their objective play experience throughout the experiment. To provide an objective measure of play experience, we collected a player's physiological data during game play. It has previously been shown that the body's physiological reaction changes when presented with a stimuli - such as a video game - and correlates strongly with the user's emotional experience [13] and in-game events [6]. We operationalize objective player experience through the measurement of two orthogonal signals galvanic skin response (GSR) to measure arousal (degree of activation), and facial electromyography (EMG) to measure valence (positive or negative). Differences in the physiological measures would reveal that players reading negative reviews experience the game differently from players reading positive reviews, while similar measures would reveal that the play experiences themselves are not different.

Similar to our previous research, our results show that players' ratings of a game are systematically affected by reading positive or negative review text of a game prior to playing it. We also show that these differences are not fully explained by subjective changes in the players' moods as a result of reading emotionally-charged text. As predicted by previous work, we found significant physiological differences for the different tasks in the experiment (i.e., reading reviews, playing game). Finally, we did not find objective differences in players' physiological responses to the game depending on the valence of the review text for either the valence or the arousal signals. These findings suggest that the variations in player ratings of the game's quality can be attributed to a cognitive rationalization of players' experiences with previously read review text, and not to differences in the play experiences themselves. Important for our understanding of the impact of reading game reviews on subsequent player experience, we have shown that despite rating the games differently, players' experience of the game was essentially the same.

\section{Related Work}

Previous work has shown that negative stimuli have a significantly greater effect on an individual than positive stimuli, an effect that has been observed in almost every area of psychology [1]. For example, it has been shown that negative information is more attention grabbing [1][4], influences impression more [4], is remembered better [16], and is cognitively processed more carefully than positive information [1], resulting in a greater biasing effect of negative stimuli. 
Persuasive power has been explored in the domains of movies [18], e-commerce [20], advertising [23], and news [19]; however, there has been little investigation into the effect on player experience. Jenkins et al. [8] studied the effects of showing a high, low, or no game score to players. The authors found when players were exposed to a high review score (e.g., 90\%) they were more likely to rate a game higher than if they were exposed to a low review score (e.g., 61\%), and attributed their results to "anchoring" [22]. Anchoring is a biasing effect where an individual's ability to estimate is biased toward a previously known value. In our previous work [10] we found that the biasing effect remained when anchoring effects were removed. $\mathrm{We}$ presented participants with various affective text stimuli including game reviews, user comments, movie reviews, and no text at all. We found that the valence of the reviews significantly affected player ratings, that the authority of the text did not matter (both user comments and critic reviews showed effects), and that the relevance of the text did matter (no differences were observed after reading positive and negative movie reviews). We also found that the biasing effect was strongest in the negative text conditions - consistent with similar work in psychology [1] - and likely primarily a cognitive effect caused by the more careful processing of the negative affective text. Finally, we showed that the ratings differences could not be explained by changes in subjectively-measured mood. This work raised the question of whether the ratings differences were a result of a change in player experience or a reflection of the player's cognitive rationalization of their experience with the review text. The present study answers this question by evaluating the effect of game reviews on objective player experience measured using physiological signals.

The study of players' physiological responses to video game stimuli has seen a significant amount of research. Mandryk et al.'s work [10,11] has explored the psychophysiological effects of games on an individual, and showed that the physiological measures were related to the subjective responses of players [13], and that the physiological measures could be used to build a mathematical model of player emotional state [12]. Drachen et al. [3] also found a significant correlation between psychophysiological arousal and self-reported player experience. Hazlett showed a relationship between players' physiological measures and in-game events. He found positive facial EMG activity to be higher during positive events, while negative facial EMG activity was greater during negative events [5,6]. Ravaja et al. [17] also showed a relationship between in-game events and phasic responses in GSR, EMG and heart rate acceleration, and Nacke et al. [14] showed that challenging game situations evoke positive affect and high arousal in players as measured by GSR and EMG. Finally, although there are significant individual differences in physiological responses, researchers have demonstrated success in the normalization of GSR [11] and EMG [6] data for between-participant comparisons. These works demonstrate the relationship between physiological responses with both self-report and in-game events. However, these studies focus on the effects of the game condition or the game itself on physiological measures. In our study, we extend these works by examining the effect of systematically-processed [2] information read prior to play on a player's physiological response. 


\section{Experiment}

\subsection{Design, Apparatus, Participants, and Procedure}

We used a single-factor between-subjects design manipulating two levels of valence (positive, negative). In our previous study we found the biasing effect manifests when the text is relevant to the task being performed [10], so we used game review text. We created the review text by modifying real reviews to ensure that the text stimuli were comparable across conditions and as authentic as possible. To create the positive and negative versions, we used affectively-charged antonyms. To control heuristic cue effects [2] the content and subject matter between conditions was maintained as much as possible. To increase participant buy-in, they were informed that the text had been taken from a professional review website. In all cases, the review text was about 1000 words. To ensure that the affective tone (positive or negative) of our text was comparable across conditions, we used the Linguistic Inquiry Word Count (LIWC) text analysis tool [15] to measure the valence of review text for each game (see [10]).

For our experiment we used a little-known game. Tag: The Power of Paint is a 3D puzzle shooter, and winner of IGF's 2009 Best Student Game. The primary factor in choosing a game was obscurity - the game had to be of professional quality, but be unknown so that users did not have prior first or second hand experience with it. All participants were unfamiliar with Tag prior to participating in the experiment.

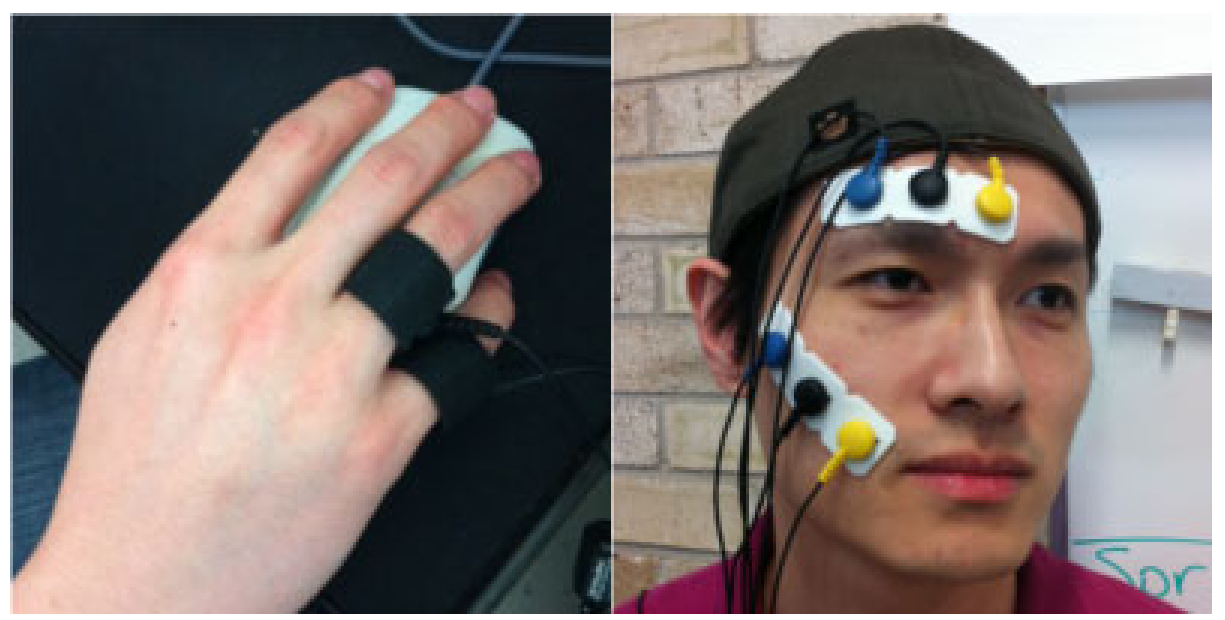

Fig. 1. Physiological sensors. GSR (left), Zygomatic and Corrugator EMG (right)

To measure physiological responses, we used Thought Technology sensors (EMG, GSR) connected to the FlexComp Infiniti encoder. The EMG band sensors were attached to the right corrugator and zygomatic muscles of the face (Figure. 1). To ensure strong EMG signals, players were screened prior to participation for excessive facial hair. The GSR sensors were attached to the ring and pinky fingers of the right hand (Figure. 1). The right hand was chosen because players moved the fingers less using the mouse with the right hand than using the keyboard with the left. 
Twenty participants ( 8 female), aged $18-25$ ( $M=21.8$ years) began by completing a consent form, and were then randomly assigned to one of the two experimental groups, balancing for sex. Players completed the experiment using a custom system developed to deploy the game and gather the survey responses using an online form.

Players were first connected to the physiological sensors and the setup was tested (for about 1 minute) to ensure strong signals from each sensor. Players then provided their demographic information. Physiological data was collected at four times during the experiment: during the baseline, during the reading of textual stimulus, while answering stimulus questions, and during gameplay. The baseline and gameplay sessions were timed to record precisely four and fifteen minutes respectively. Players were not time-limited during the reading or answering phases, rather markers were added to the data when the player started reading, stopped reading, started answering, and stopped answering. During the baseline phase, players were shown a gray screen with a cross displayed slightly off center. The player then performed a pre-text valence and arousal rating via the self-assessment mannequin (SAM) [9]. Next, participants read the text stimulus corresponding to their test condition and answered the required questions that followed. The SAM valence and arousal was measured again, and then the game was played for 15 minutes. Afterwards, the SAM valence and arousal ratings were collected a final time.

To evaluate their perception of game quality, players wrote a short game review and provided a game rating. Players were never shown numerical scores to ensure that no biasing anchoring effects were present. To help players choose scores, and to aim for consistency across players in their interpretation of the ratings scale, a guideline was provided (0-19: I hated it; 20-49: I didn't like this; 50-74: It was ok, but I'm rather indifferent; 75-89: It was good, but room for improvement; 90-100: I loved it).

\subsection{Data Processing and Analysis}

Physiological data were exported from BioGraph as raw CSV files and imported into MATLAB for processing and analysis. Data were divided based on the action being performed. Subsequent analyses were conducted during reading of the stimulus text and playing the game, although the baseline phase was used for signal normalization. EMG and GSR require different signal processing, so custom scripts were developed for each physiological sensor.

Electromyography (EMG). The raw data collected from both EMG sensors was the RMS of the signal. Consistent with normal EMG signal analysis [21] the raw data was first filtered, and then smoothed. All EMG data was subjected to a $3^{\text {rd }}$ order Butterworth low-pass filter at $500 \mathrm{~Hz}$, then smoothed using a moving average window of $0.5 \mathrm{~s}(1024 \mathrm{~Hz})$. The remaining data points were normalized by subtracting the baseline or reading values (whichever were lower) from each sample and representing each sample as a percentage using the min and max values found across all filtered and smoothed samples for a specific participant. Normalizing was required to allow for meaningful between-participant comparisons.

An EMG ratio was also calculated using a similar approach to the one described by Hazlett [6]. We calculated the total time spent in activation higher than one standard deviation above the mean for both the corrugator and zygomatic muscles. The ratio of 
zygomatic to corrugator activation was expressed as a decimal value. These ratios could safely be compared between participants.

Galvanic skin response (GSR). Similar to the EMG data processing, GSR values need to be normalized to allow for meaningful statistical comparisons between participants. GSR was normalized by calculating each sample as a percentage of the entire span of GSR, using the min and max values over all samples for one participant. This practice is consistent with GSR analysis of gameplay experience [11]. The mean and standard deviation for each GSR sample was calculated and recorded as a percentage.

Data Analysis and Dependent Measures. Our subjective dependent measures are SAM arousal, SAM valence, and game ratings. Our objective dependent measures are normalized GSR, normalized zygomatic and corrugator EMG, and Hazlett EMG emotion ratio. The subjective dependent measures do not meet the assumptions of normality and equal variance, which is typical with ratings data. We used the MannWhitney Test for all between-groups tests, and the Wilcoxon Signed Ranks Test for within-player comparisons [7]. The objective physiological measures were analyzed using the appropriate parametric statistical tests.

Table 1. Means and SDs of dependent measures for each phase, split by group. Arousal and valence were measured after the phase on a 9-pt scale, while the physiological measures were averaged over the phase and normalized between $0-1$.

\begin{tabular}{|c|c|c|c|c|c|c|}
\hline & \multicolumn{3}{|c|}{ Positive Review } & \multicolumn{3}{c|}{ Negative Review } \\
\hline & Baseline & Reading & Playing & Baseline & Reading & Playing \\
\hline Ratings & & & $80.9(8.61)$ & & & $44.3(22.94)$ \\
\hline Arousal & $2.9(0.88)$ & $3.3(1.77)$ & $6.1(1.66)$ & $2.6(0.70)$ & $2.7(1.06)$ & $3.9(1.85)$ \\
\hline Valence & $6.2(1.55)$ & $6.2(1.62)$ & $7.1(1.45)$ & $7.0(0.94)$ & $5.8(1.32)$ & $6.1(2.33)$ \\
\hline EMG Cheek & & $0.05(0.06)$ & $0.09(0.09)$ & & $0.02(0.04)$ & $0.11(0.07)$ \\
\hline EMG Brow & & $0.04(0.03)$ & $0.01(0.03)$ & & $0.02(0.03)$ & $0.02(0.02)$ \\
\hline GSR & & $0.23(0.13)$ & $0.65(0.08)$ & & $0.23(0.20)$ & $0.67(0.07)$ \\
\hline EMG Ratio & & $1.36(0.86)$ & $1.67(0.93)$ & & $1.03(0.53)$ & $2.10(1.47)$ \\
\hline
\end{tabular}

\subsection{Results}

Does the text's valence affect perception of game quality? Consistent with our findings from our previous study, players who read positive text rated the game significantly higher than players who read negative review text $(Z=3.6 p \approx .000)$.

Can the ratings differences be explained by changes in mood? Consistent with our previous work, we were interested in whether the game ratings differences could be explained by mediating changes in a player's mood. Perhaps the players reading positive text were put into a more positive mood and then enjoyed the game more than players reading negative text (thus rating it higher). We asked players to rate their valence and arousal three times: prior to reading the game review, after reading the game review, and after playing the game. Means and variances are in Table 1. 
There were no differences between the two groups for any of the three valence ratings $(Z(p)$ - Pre-read: 1.07(.315); Post-read: .70(.529); Post-play: .97(.353)). There were also no differences in arousal between the groups prior to or after reading the game reviews $(\mathrm{Z}(\mathrm{p})$ - Pre-read: .80(.481); Post-read: .82(.481)). However, there was a difference in the arousal rating after playing the game, where the positive review group rated their post-game arousal significantly higher than the negative review group ( $\mathrm{Z}(\mathrm{p})$ - Post-play: 12.45(.015)). To determine what this difference means, we looked into arousal changes over the experiment for each group. There were two arousal differences that we considered - the difference between before and after reading the review text and the difference between after reading review text and after play. For the negative group, there was no difference in arousal after reading the review text $(\mathrm{Z}=.58, \mathrm{p}=.564)$ or after play $(\mathrm{Z}=1.78, \mathrm{p}=.076)$. For the positive group, arousal did not increase after reading the review $(\mathrm{Z}=.96, \mathrm{p}=.336)$, but did increase after playing the game $(\mathrm{Z}=2.68, \mathrm{p}=.007)$. Thus it appears that reading the review text did not increase players' arousal for either group; however, playing the game significantly increased the positive group's arousal, but only marginally increased the negative group's arousal, resulting in a net difference between the groups.

\section{Are the ratings differences reflected in objectively-measured player experience?}

The main goal of the present study was to determine whether the game ratings differences are reflected in differences in player experience during play, or whether the differences are a cognitive rationalization of similar play experiences with differing review texts. We conducted a RM-MANOVA with one between-subjects factor (valence of review - positive or negative) and one within-subjects factor (phase of experiment - reading review or playing game) on four dependent measures (GSR, EMG cheek, EMG brow, EMG ratio). Means and variances are shown in Table 1.

As expected, there was a significant main effect of phase on GSR $\left(\mathrm{F}_{1,18}=143.6\right.$, $\left.\mathrm{p} \approx .000, \eta^{2}=.89\right)$, EMG cheek $\left(\mathrm{F}_{1,18}=15.0, \mathrm{p}=.001, \eta^{2}=.46\right)$, and EMG ratio $\left(\mathrm{F}_{1,18}=6.2\right.$, $\mathrm{p}=.023, \eta^{2}=.26$ ), which showed that players were more aroused and experienced higher valence during game play than during reading. There was no effect of phase on EMG brow $\left(\mathrm{F}_{1,18}=1.0, \mathrm{p}=.329, \eta^{2}=.05\right)$. These results confirm our expectations that gameplay is more exciting than reading review text, and also demonstrate the capability of the physiological signals to differentiate player experience objectively.

There was no main effect of valence of review text on any of the dependent measures (GSR: $F_{1,18}=.03, p=.879, \eta^{2} \approx .00 ;$ EMG cheek: $F_{1,18}=.08, p=.780, \eta^{2} \approx .00$; EMG brow: $\mathrm{F}_{1,18}=.75, \mathrm{p}=.399, \eta^{2}=.04$; EMG ratio: $\left.\mathrm{F}_{1,18}=.02, \mathrm{p}=.885, \eta^{2} \approx .00\right)$. These results show that there were no overall objective differences between the two groups.

There were also no interaction effects between valence group and phase on any of the dependent measures (GSR: $\mathrm{F}_{1,18}=.021, \mathrm{p}=.886, \eta^{2} \approx .00$; EMG cheek: $\mathrm{F}_{1,18}=2.0$, $\mathrm{p}=.175, \eta^{2}=.10$; EMG brow: $\mathrm{F}_{1,18}=1.9, \mathrm{p}=.184, \eta^{2}=.10$; EMG ratio: $\mathrm{F}_{1,18}=1.9, \mathrm{p}=.187$, $\left.\eta^{2}=.10\right)$. These results show that the two groups did not respond differently during the two phases. We may have expected similar levels of objective experience during reading, and that the positive group might demonstrate more enjoyment during play than the negative group. However, this is not the case as our results suggest that there are no differences in the responses of the two groups to the experiment. 


\section{Discussion}

There are four main results to take away from our findings:

1. Reading positive or negative game reviews influences subsequent player ratings of game quality.

2. The ratings differences cannot be explained by changes in the self-reported player valence, but may have been mediated by player arousal. Increases in selfreported arousal after playing were significant for players who read positive reviews, and marginal for players who read negative reviews, resulting in a between-group difference in self-reported arousal post play.

3. There were significant increases in GSR, EMG of the cheek, and the Hazlett EMG ratio when playing the game over reading the review text, demonstrating the sensitivity of these signals to measure player experience.

4. There were no differences in any of the physiological measures between those who read positive or negative reviews, suggesting that player experience did not change as a result of reading review text, but that ratings differences were a postplay rationalization of experience with the previously-read reviews.

The first three results are consistent with previous work, while the fourth resolves a question left unanswered by previous work. We focus our discussion on the contributions of game-related pre-play and play experiences on resulting opinion about a game, the potential impact of our results on industry practices, and the research opportunities in this space.

\subsection{Effects of Review Text on Player Experience}

We conducted this work to shed light on an unanswered question - does reading positive or negative game reviews affect player experience? Previous work had shown that reading positive or negative game reviews affected player perception of game quality provided by a game rating, and showed that these differences were not mediated by changes in a player's mood [10]. Our work replicated these results, but also showed that reading positive or negative game reviews did not affect player experience during play of the game itself. So if players who read positive text did not experience the game differently than those who read negative text, why did they rate the game significantly higher?

We feel that the game ratings are not indicative solely of the player's experience, but are actually a cognitive rationalization of the experience in the context of the previously-read review text. This does not mean that players are under a false impression of their experience, or that review text wins when there is a discrepancy between the review content and their experience. It is possible, and quite likely, that players who read negative text would subjectively characterize their experience as worse than players who read positive text, indicating that the rationalization of experience with the influencing reviews affected not only the perception of game quality, but also the perception of the play experience. That measured player experience did not differ does not make the reflective difference in experience any less real to a player or any less true. It does mean that players who read negative reviews decided that they liked the game less than those who read positive reviews, 
not because of differences in play experience, but in spite of no differences in play experience. This lack of difference in play experience after reading game reviews has important impact for the game industry.

\subsection{Impact on Industry}

Current review methods used in the game industry include game reviews from both professional and amateur critics, and discussion forums where players can discuss their opinions and experiences. These textual channels offer players a freely available source of influential reports, which then influence player perception of game quality. The game industry is known for releasing games to critics prior to release. As our results show that reading reviews (especially negative reviews) biases the player's perception of the game, game studios should be careful of critical receptions prior to game release. It is tempting to believe that a good play experience will outweigh negative buzz surrounding a game, but we show that this may not be the case. Even though experience of the game was not different, players who read negative reviews were still more critical in their perception of the game's quality.

Our findings can also be extended to other marketplaces where the exposure of reviews and ratings is becoming more prominent. For example, the Apple app store prominently displays user ratings and comments right on the main app page for each app. Our results suggest that these ratings may adversely affect the perception of quality of those products for users who read the reviews prior to using the app. Although these ratings often come from users of the apps and not professional critics, our previous work revealed that the authority of the review source did not matter; players were influenced both by game reviews and by user comments. Developers need to be aware of the impact these reviews and comments have, not only directly on their sales, but also on the experience users have with their products.

In an online community this negative effect can snowball as users who are influenced by critic reviews may in turn post negative comments online, influencing an even larger user population. As this cycle continues with new users reading and being influenced by the negative press, the bottom line of a game or an app in terms of commercial success could be negatively affected.

\subsection{Alternate Explanations of the Results}

There are cognitive biases that can influence a user's perception of a game quality. We controlled for related biases in our study in order to attribute the results to the reading of review text and not other cognitive biases. For example, affective priming uses a stimulus, unrelated to the task or object to be viewed, to change a participant's affective state or mood [23]. Because our review text was related to the game, we can assume that our results are not due to affective priming. Anchoring is a biasing effect where an individual's ability to estimate is biased toward a previously-known value. In previous work, players exposed to a negative rating of a game rated that game lower themselves [8]. The authors identify this as an effect of anchoring. In our study, we did not present the players with any numerical ratings, so the content and tone of the review text was causing the difference in perception of game quality. Thus, our results cannot be attributed to the biasing effects of anchoring or affective priming. 
Although players rated the game differently depending on the valence of previously-read review text, we observed no physiological differences between the groups. One might suggest that there were in fact differences in player experience, but that physiological measures are not sensitive enough to show differences. Because our results showed significant and predictable physiological differences between reading the review text and playing the game, we feel that the signals are a good measure of player experience and are sensitive enough to reveal differences.

\subsection{Limitations and Future Work}

We examined average physiological responses over a play session, and did not test phasic responses to specific game events. Our results confirm that player experience was not different overall, but cannot confirm whether player responses within a play session were similar. It is possible that the two valence groups responded differently to specific game events (especially those that were targeted in the game reviews), but that these differences were not large enough in magnitude or frequency to affect average experience. Future work examining phasic responses to in-game events would help to shed light on the player reactions that make up overall experience.

We gathered subjective reactions through game quality ratings. It is possible that although we saw no objective differences in play experience, the interpretation of similar play experiences in the context of different review text might cause players to subjectively characterize their play experience differently. Although outside the scope of this work, it would be interesting to know whether the cognitive rationalization of play experience with review text resulting in differing game ratings would also be reflected in differing subjective characterization of play experience.

Our study is the first use of physiological measures to evaluate experience prior to the playing of a game, which is important when considering biasing effects that can influence experience. The importance of pre-play events should not be dismissed, as we show that the experience begins before engaging in actual play. This introduces a variety of interesting research questions as events prior to play deserve consideration, and more study is required to determine their impact on player experience. Our study only looks at biasing effects of game review text, but our methods could prove useful in the evaluation of any number of pre-play activities, such as the effects of any pregame menu traversal, loading times, or profile creation.

\section{Conclusion}

In this paper we have demonstrated the biasing effect that reading video game reviews has on player perceptions of a game. We show that these effects cannot be entirely explained by mediating changes in mood, and that the effects do not influence player experience as measured objectively through physiological sensors. Our major contribution is showing that the differences in perception of game quality that result from playing a game after reading a review are not stemming from differences in the play experience, but are a cognitive rationalization of the experience with the content and tone of the review. Our results are important for understanding player experience and for the game industry where reviews and commenting forums affect sales.

Acknowledgments. We thank NSERC and the GRAND NCE for funding. 


\section{References}

1. Baumeister, R.F., et al.: Bad is stronger than good. Review of General Psychology 4, 323-370 (2001)

2. Chaiken, S., Eagly, A.H.: Heuristic and Systematic Information Processing within and. Unintended Thought 212 (1989)

3. Drachen, A., et al.: Correlation between heart rate, electrodermal activity and player experience in first-person shooter games. In: Proceedings of the 5th ACM SIGGRAPH Symposium on Video Games, pp. 49-54. ACM, New York (2010)

4. Fiske, S.T.: Attention and weight in person perception: The impact of negative and extreme behavior. Journal of Personality and Social Psychology 38(6), 889-906 (1980)

5. Hazlett, R.L.: Measuring emotional valence during interactive experiences: boys at video game play. In: Proceedings of the SIGCHI Conference on Human Factors in Computing Systems, pp. 1023-1026 (2006)

6. Hazlett, R.: Using Biometric Measurement to Help Develop Emotionally Compelling Games. In: Game Usability: Advancing the Player Experience, pp. 187-205. Morgan Kaufmann, San Francisco (2008)

7. Huck, S.W., Cormier, W.H.: Reading statistics and research. HarperCollins, New York (1996)

8. Jenkins, R., et al.: The Influence of Professional Critic Reviews. EEDAR/SMU Behavioral Study. Southern Methodist University, Guildhall. Electronic Entertainment Design and Research (2010)

9. Lang, P.: Behavioral treatment and bio-behavioral assessment: Computer applications. In: Technology in Mental Health Care Delivery Systems, pp. 119-137. Ablex Pub. Corp., Norwood (1980)

10. Livingston, I.J., et al.: The Impact of Negative Game Reviews and User Comments on Player Experience. Presented at the Sandbox 2011: The 6th ACM SIGGRAPH, Vancouver, Canada (2011)

11. Mandryk, R.L.: Physiological Measures for Game Evaluation. In: Game Usability: Advice from the Experts for Advancing the Player Experience, Morgan Kaufmann, San Francisco (2008)

12. Mandryk, R.L., et al.: A continuous and objective evaluation of emotional experience with interactive play environments. In: Proceedings of the SIGCHI Conference on Human Factors in Computing Systems, pp. 1027-1036. ACM, Montréal (2006)

13. Mandryk, R.L., et al.: Using psychophysiological techniques to measure user experience with entertainment technologies. Behaviour \& Information Technology 25(2), 141 (2006)

14. Nacke, L.E., Lindley, C.A.: Affective Ludology, Flow and Immersion in a First-Person Shooter: Measurement of Player Experience (2010)

15. Pennebaker, J.W., et al.: LIWC, http: / /www. Iiwc.net/

16. Pratto, F., John, O.P.: Automatic Vigilance: The Aention-Grabbing Power of negative Social Information. Social Cognition: Key Readings 250 (2005)

17. Ravaja, N., et al.: Phasic emotional reactions to video game events: A psychophysiological investigation. Media Psychology 8(4), 343-367 (2006)

18. Reinstein, D.A., Snyder, C.M.: The Influence of Expert Reviews on Consumer Demand for Experience Goods: A Case Study of Movie Critics. The Journal of Industrial Economics 53(1), 27-51 (2005)

19. Sundar, S.S., et al.: News cues: Information scent and cognitive heuristics. Journal of the American Society for Information Science and Technology 58(3), 366-378 (2007)

20. Sundar, S.S., et al.: Authority vs. peer: how interface cues influence users. In: Proceedings of the 27th International Conference Extended Abstracts on Human Factors in Computing Systems, pp. 4231-4236 (2009) 
21. Tassinary, L.G., Cacioppo, J.T.: The skeletomotor system: Surface electromyography. Handbook of Psychophysiology 2, 163-199 (2000)

22. Tversky, A.K., Kahneman, B.: Judgment under uncertainty: Heuristics and biases. Science 185(4754), 1124-1131 (1974)

23. Yi, Y.: Cognitive and affective priming effects of the context for print advertisements. Journal of Advertising 19(2), 40-48 (1990) 